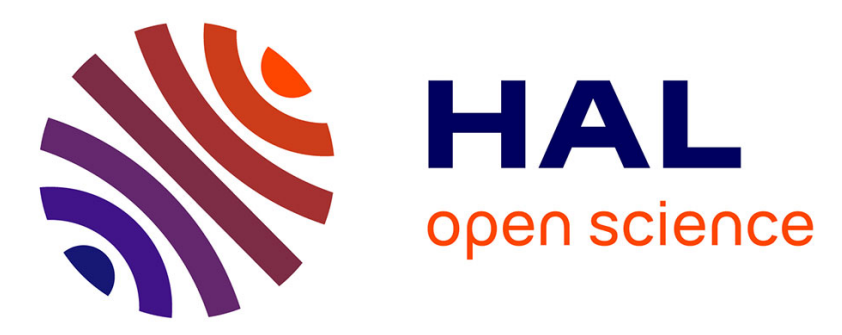

\title{
Signature of gate-tunable magnetism in graphene grafted with Pt-porphyrins
}

Chuan Li, Katsuyoshi Komatsu, S Bertrand, G Clavé, S Campidelli, A Filoramo, S Guéron, H Bouchiat

\section{To cite this version:}

Chuan Li, Katsuyoshi Komatsu, S Bertrand, G Clavé, S Campidelli, et al.. Signature of gate-tunable magnetism in graphene grafted with Pt-porphyrins. Physical Review B: Condensed Matter and Materials Physics (1998-2015), 2016, 93, pp.045403. 10.1103/PhysRevB.93.045403 . cea-01253564

\section{HAL Id: cea-01253564 https://hal-cea.archives-ouvertes.fr/cea-01253564}

Submitted on 11 Jan 2016

HAL is a multi-disciplinary open access archive for the deposit and dissemination of scientific research documents, whether they are published or not. The documents may come from teaching and research institutions in France or abroad, or from public or private research centers.
L'archive ouverte pluridisciplinaire HAL, est destinée au dépôt et à la diffusion de documents scientifiques de niveau recherche, publiés ou non, émanant des établissements d'enseignement et de recherche français ou étrangers, des laboratoires publics ou privés. 


\title{
Signature of gate-tunable magnetism in graphene grafted with Pt-porphyrins
}

\author{
Chuan Li, ${ }_{1}^{1}$ Katsuyoshi Komatsu, ${ }^{1}$ S. Bertrand, ${ }^{1}$ G. Clavé, ${ }^{2}$ S. Campidelli, ${ }^{2}$ A. Filoramo, ${ }^{2}$ S. Guéron, ${ }^{1}$ and H. Bouchiat ${ }^{1}$ \\ ${ }^{1}$ Laboratoire de Physique des Solides, CNRS, Univ. Paris-Sud, Université Paris-Saclay, 91405 Orsay Cedex, France \\ ${ }^{2}$ CEA Saclay, IRAMIS, NIMBE (UMR 3685), Laboratoire d'Innovation en Chimie des Surfaces et Nanosciences (LICSEN), \\ F-91191 Gif sur Yvette, France
}

(Received 14 May 2015; revised manuscript received 18 November 2015; published 5 January 2016)

\begin{abstract}
Inducing magnetism in graphene holds great promises, such as controlling the exchange interaction with a gate electrode, and generating exotic magnetic phases. Coating graphene with magnetic molecules or atoms has so far mostly led to decreased graphene mobility. In the present work, we show that Pt-porphyrin molecules adsorbed on graphene lead both to an enhanced mobility, and to gate-dependent magnetism. We report that porphyrins can act both as donor or acceptor molecules, depending on the initial doping of the graphene sheet. The porphyrins transfer charge and ionize around the charged impurities on graphene, and, consequently, the graphene doping is decreased and its mobility is enhanced. In addition, ionized porphyrin molecules carry a magnetic moment. Using the sensitivity of mesoscopic transport to magnetism, in particular, the superconducting proximity effect and conductance fluctuations, we explore the magnetic order induced in graphene by the interacting magnetic moments of the ionized porphyrin molecules. Among the signatures of magnetism, we find two-terminal-magnetoresistance fluctuations with an odd component, a tell-tale sign of time-reversal symmetry breaking at zero field, which does not exist in uncoated graphene samples. When graphene is connected to superconducting electrodes, the induced magnetism leads to a gate-voltage-dependent suppression of the supercurrent, modified magnetic interference patterns, and gate-voltage-dependent magnetic hysteresis. The magnetic signatures are greatest for long superconductor/graphene/superconductor junctions, and for samples with the highest initial doping, compatible with a greater number of ionized, and thus magnetic porphyrin molecules. Our findings suggest that long-range (of the order of the coherence length, or micrometers) magnetism is induced through graphene by the ionized porphyrins' magnetic moment. This magnetic interaction is controled by the density of carriers in graphene, a tunability that could be exploited in spintronic applications.
\end{abstract}

DOI: 10.1103/PhysRevB.93.045403

\section{INTRODUCTION}

Because of its conical band structure and the possibility to continuously tune the Fermi level with a gate voltage, graphene has opened a broad new field of investigation of 2D electronic transport. At low temperature, the phase coherence length, of the order of one micrometer, offers the possibility to explore mesoscopic aspects of transport in graphene such as conductance fluctuations and proximity-induced superconductivity. More than classical transport, quantum transport is especially sensitive to the nature of scattering impurities on graphene, in particular, to their charged, neutral, or polar character [1]. The spatial extent of the scattering potential also plays an important role: short-range neutral scatterers cause intervalley scattering, in contrast to long-range charged impurities. Beyond the characterization of intrinsic impurities of graphene, it is tempting to add specific impurities to induce new functionalities and tune them in a controlled way. Adsorbates on graphene transistors have, for instance, been shown to affect the transport characteristics of graphene-based sensors [2]. On a more fundamental level, a longstanding goal is to induce and control physical properties involving spin degrees of freedom such as spin-orbit coupling and magnetism. Specific signatures of spin-orbit or magnetic scatterers are expected, whose effect should be tunable with the gate voltage [3-6]. To reach this goal, graphene has been coated with magnetic (transition metal or rare earth) atoms $[7,8]$ as well as molecular magnets, with mixed results. Adsorbed magnetic atoms reduce graphene's mobility, with no clear concurrent magnetic signature. In contrast, the magnetization reversal of molecular magnets has been detected in a graphene nanoconstriction [9]. Possible signatures of enhanced spinorbit interactions have also been observed in graphene coated with small nonmagnetic metallic clusters [10]. Magnetism could also be induced by creating vacancies in graphene [11,12] or adsorbing atomic hydrogen [13], with a doping-dependent magnetic signature. However, in both cases, this magnetism is induced at the cost of an unavoidable decrease of sample mobility.

In the present work, we functionalize graphene with a thin film of Pt-porphyrins. The first layer directly in contact with graphene can interact with graphene's delocalised $s p^{2}$ orbitals and is expected to form an ordered array [14]. Neutral Pt-porphyrins are nonmagnetic, but the ionized form carries a magnetic moment of one Bohr magneton [15]. At room temperature, we find that the molecules dope the graphene, demonstrating that charge transfer occurs. Either electrons or holes can be transferred to the graphene layer, depending on its initial doping. More surprisingly, the graphene's mobility increases upon molecules deposition. Using the high sensitivity of mesoscopic transport, in particular the superconducting proximity effect and conductance fluctuations [16], we show evidence of long-range magnetism induced in several porphyrin-coated samples. This magnetism leads to field asymmetry of conductance fluctuations in samples with normal electrodes, and suppression of supercurrent or modified Fraunhoffer patterns in samples with superconducting electrodes. The signatures are largest for samples with high initial doping, for which more porphyrins ionize and thus become magnetic.

One implication of these findings is that it is possible to control the number of magnetic porphyrins by their degree 
of ionization. In all experiments, this magnetism is found to depend on the gate voltage, a tunability that could be exploited in spintronic devices. These findings also constitute evidence for Fermi-level controlled exchange interaction between localized spins and graphene.

\section{SAMPLE PREPARATION}

The samples are prepared by exfoliation of high-quality graphite and deposition onto an oxidized doped silicon wafer (acting as a backgate). Monolayers are selected by optical microscopy. The metallic contacts are made by electron beam lithography followed by deposition of different metallic bilayers, $\mathrm{Ti} / \mathrm{Au}, \mathrm{Ti} / \mathrm{Al}$, or $\mathrm{Pd} / \mathrm{Nb}$. All samples were measured at room temperature before deposition of the molecules. The Pt-porphyrin, see Fig. 1, contains a Pt atom at the center of the characteristic cyclic organic cage of four pyrrole subunits interconnected via methine bridges $(=\mathrm{CH})$. The porphyrins were prepared as described in [17]. The electronic structure of porphyrins, as determined by optical absorption measurements

(a)

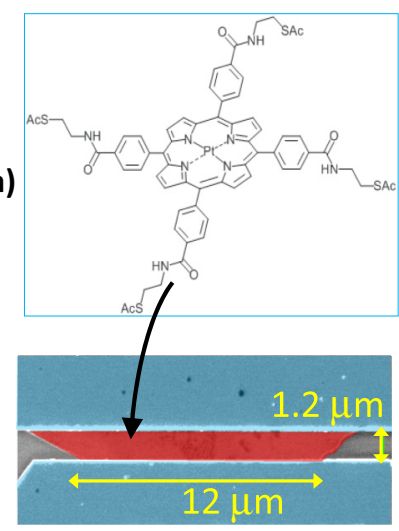

(b)
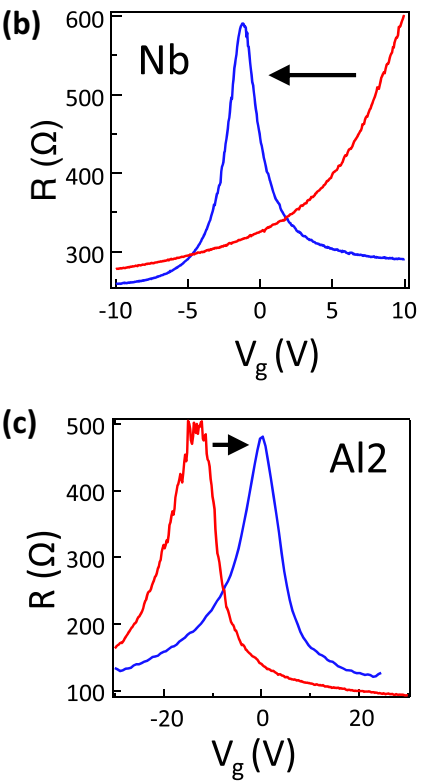

FIG. 1. Charge transfer at room temperature. (a) Electron microscope image of the graphene sample connected to $\mathrm{Pd} / \mathrm{Nb}$ electrodes, and representation of the Pt-porphyrin. (b) and (c) Gate dependence of resistance before and after porphyrins deposition for two samples, $\mathrm{Nb}$ ( $\mathrm{Pd} / \mathrm{Nb}$ electrodes), and $\mathrm{Al} 2$ (Ti/Al electrodes). Without porphyrins (red curves), the samples can be hole-doped [Dirac Point initially at positive gate voltage (b)] or electron-doped (c). After grafting porphyrins, in both cases, the Dirac point of graphene is brought to practically zero: graphene has become neutral. This implies that charge transfer occurs between graphene and porphyrins and the molecules can be donors (b) as well as acceptors (c) of electrons. We believe that this neutralization of the charged defects in graphene occurs because it reduces the electrostatic energy of the system thanks to a better spatial confinement than when charged impurities are screened by graphene's carriers [since that screening occurs over larger distances, of the order of the Thomas-Fermi screening length $(10$ to $30 \mathrm{~nm})$ ]. This charge transfer is accompanied by gate voltage hysteresis and relaxation effects (see Fig. 2).

TABLE I. Principal characteristics of the samples cooled down after deposition of porphyrins. (Except control samples Au3 and Al1 for which no porphyrins were deposited.) The mobility $\mu$ was measured at room temperature near the Dirac point before porphyrin deposition. $\Delta V_{D}$ is the shift in gate voltage of the Dirac point after deposition of the porphyrins. This number gives an indication of the concentration of ionized porphyrins: a shift of 10 to $40 \mathrm{~V}$ in Dirac point upon porphyrin deposition corresponds to a transfer of 1 to $410^{12}$ charges per $\mathrm{cm}^{2}$, implying that $4 \%$ to $15 \%$ of the porphyrin molecules in contact with graphene ionize.

\begin{tabular}{lccccc}
\hline \hline Sample & Contact & $\begin{array}{c}\text { Length } \\
(\mu \mathrm{m})\end{array}$ & $\begin{array}{c}\text { width } \\
(\mu \mathrm{m})\end{array}$ & $\mu\left(\mathrm{cm}^{2} \mathrm{~V}^{-1} \mathrm{~s}^{-1}\right)$ & $\Delta V_{D}(\mathrm{~V})$ \\
\hline $\mathrm{Au} 1$ & $\mathrm{Ti} / \mathrm{Au}$ & 0.8 & 3.5 & 2000 & -10 \\
$\mathrm{Au} 2$ & $\mathrm{Ti} / \mathrm{Au}$ & 0.7 & 8 & 1400 & $\leqslant-20$ \\
$\mathrm{Au} 3$ & $\mathrm{Ti} / \mathrm{Au}$ & 0.8 & 3.5 & 1600 & no porph. \\
$\mathrm{Nb}$ & $\mathrm{Pd} / \mathrm{Nb}$ & 1.2 & 12 & 5000 & -10 \\
$\mathrm{Al1}$ & $\mathrm{Ti} / \mathrm{Al}$ & 0.5 & 3.4 & 3000 & no porph. \\
$\mathrm{Al} 2$ & $\mathrm{Ti} / \mathrm{Al}$ & 0.45 & 4 & 2000 & 15 \\
$\mathrm{Al3}$ & $\mathrm{Ti} / \mathrm{Al}$ & 0.5 & 4 & 2000 & 15 \\
$\mathrm{Ald} 1$ & $\mathrm{Ti} / \mathrm{Al}$ & 0.4 & 2.6 & 150 & $\geqslant 40$ \\
$\mathrm{Ald} 2$ & $\mathrm{Ti} / \mathrm{Al}$ & 0.6 & 4 & $\leqslant 100$ & $\geqslant 30$ \\
\hline \hline
\end{tabular}

and scanning tunneling spectroscopy, is characterized by a HOMO (highest occupied molecular orbital) -LUMO (lowest unoccupied molecular orbital) gap of the order of $2 \mathrm{eV}$ [18]. We deposited Pt porphyrins at room temperature according to the following protocol. We first checked that deposition of the sole solvent tetrahydrofuran (THF) does not modify the gate voltage dependence of the sample's conductance. We then deposited a $10 \mu l$ drop of a $1 \mathrm{mM}$ solution of Pt porphyrins in THF. This corresponds to a few hundred layers of porphyrins covering the graphene after evaporation of the THF solvant. We have changed this number of layers by a factor 10 and find that the low-temperature results (that demonstrate the gatedependent magnetism) do not depend on the layer thickness, consistent with a porphyrin/graphene interaction restricted to the first layer. We have functionalized over twenty graphene samples. Among those samples, ten were investigated at low temperature both before and after functionalization, and their characteristics are detailed in Table I.

\section{CHARGE TRANSFER BETWEEN GRAPHENE AND PORPHYRINS: NEUTRALIZATION OF GRAPHENE BY PORPHYRINS}

At room temperature (RT), we systematically measured the gate voltage dependence of the resistance before and after porphyrin deposition. A striking neutralization effect, shown on Fig. 1, was observed on all investigated samples: the Dirac point of all samples is shifted to nearly zero gate voltage after deposition. This means not only that charges are transferred between porphyrins and graphene, but also that porphyrins can be both electron donors or acceptors. Since the HOMO-LUMO gap of the porphyrin $(2 \mathrm{eV})$ is much greater than the typical Fermi energy differences between the graphene samples we have investigated $(0.1 \mathrm{eV})$, this implies a local pinning of the HOMO (LUMO) level of the porphyrins to the hole (electron) doped Fermi level of graphene. Electron transfer has already 

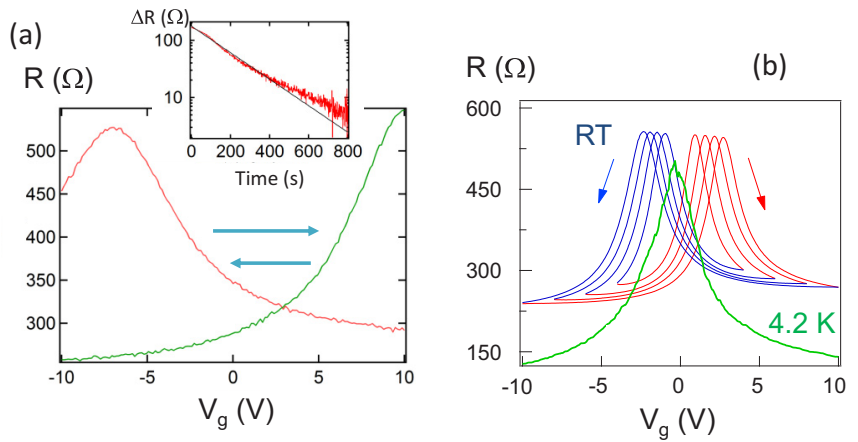

FIG. 2. Hysteresis in gate dependence at room temperature of the porphyrin-coated $\mathrm{Pd} / \mathrm{Nb}$ sample. (a) Modification of the Dirac point position after keeping the sample for one hour at $V_{G}= \pm 10 \mathrm{~V}$. (Inset) Time evolution of the sample resistance at fixed gate voltage after a rapid gate shift from -10 to $+10 \mathrm{~V}$. The slow relaxation can be fitted by $\Delta R=R_{0} e^{-t / t_{0}}$ with $R_{0}=165 \Omega$ and $t_{0}=187 \mathrm{~s}$. (b) Gate dependent resistance at room temperature for different excursions. One can note the hysteresis, which amplitude depends on the gate bias excursion in the presence of porphyrins. Bottom (green) curve: gate-dependent resistance measured at $4.2 \mathrm{~K}$. The hysteresis is absent at low temperature. The vertical shift to lower resistance is due to the superconductivity of the $\mathrm{Pd} / \mathrm{Nb}$ electrodes.

been reported for highly hole-doped porphyrin-grafted carbon nanotubes [19,20], and, more recently, for highly hole-doped Zn-porphyrins-grafted graphene [21]. However, the possibility to also inject holes in graphene with porphyrins has, to our knowledge, not previously been demonstrated.

At room temperature the gate dependence of the resistance is hysteretic, with a slow exponential relaxation of the sample's resistance in response to a fast gate voltage change. We relate the RT hysteresis and slow relaxation to hopping processes through neighboring molecules [22,23], leading to a slow (hundred second time scale) charge transfer across porphyrin layers above the graphene. Figure 2 illustrates how the Dirac point is shifted to $-V_{0}$, with a broadened $R\left(V_{g}\right)$ curve, after keeping the sample at a nonzero gate voltage $V_{0}$ at room temperature. This phenomenon can be seen as temporary, artificial doping of graphene by the transfer and storage of charges in the molecules. Similar effects have been observed with graphene samples functionalized with insulating nanoparticles (iron and titanium oxide, $\mathrm{CdSe}$ ) showing evidence of charge neutralization of graphene, mobility increase, and gate voltage dependent charge transfer between graphene and nanoparticles [24].

When cooling the sample at zero gate voltage, the Dirac point stays unchanged close to $V_{g}=0$. In contrast to the RT behavior, there is no gate-dependent charge transfer at low temperature (below $4.2 \mathrm{~K}$ ). This is demonstrated by magnetoresistance measurements in the quantum Hall regime, for which the carrier density $n_{c}$, estimated with the same parameters as for the bare graphene sample (same capacitance, etc.) yields the quantum Hall plateaus for the coated sample at exactly the same filling factors as before, see Fig. 3. Another important feature is the increase in sharpness of the $R\left(V_{g}\right)$ curve after porphyrin deposition, with a higher resistance at the charge neutrality point (Dirac point) [see Fig. 3(a)], implying that coating with Pt-porphyrins results in a higher (a)

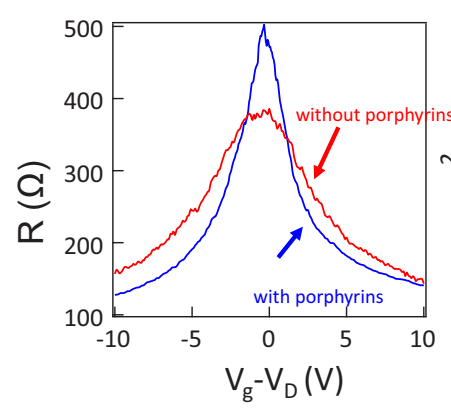

(b) $\quad \mathrm{V}_{\mathrm{g}}-\mathrm{V}_{\mathrm{D}}(\mathrm{V})$

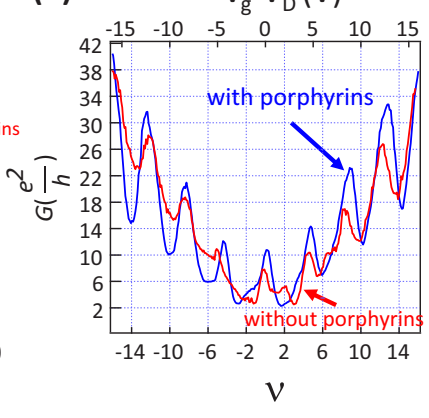

FIG. 3. Improvement of the sample quality after porphyrins deposition ( $\mathrm{Pd} / \mathrm{Nb}$ sample). (a) Zero magnetic field gate voltage sweep of two wire resistance at $4.2 \mathrm{~K}$, before (red) and after (blue) deposition of Pt-porphyrins. The origin is taken at the Dirac point $V_{D}$ for both curves. The sharper curve with a higher Dirac peak demonstrates that the sample quality has improved thanks to porphyrin coating, with a mobility increase of $20 \%$. (b) Two-wire measurement of the resistance in the quantum Hall regime, at $100 \mathrm{mK}$ and in a perpendicular magnetic field of $B=5 \mathrm{~T}$ (for which the $\mathrm{Nb}$ electrodes are nonsuperconducting), before (red curve) and after (blue curve) grafting. The gate voltage is expressed in terms of the filling factor $v=n_{c} \phi_{0} / B$, with the charge density $n_{c}$ computed assuming that only the backgate charges the graphene. The Hall plateaus (visible even in a two wire measurement) are better defined, confirming the higher sample quality. The gate voltage position of the plateaus is unchanged, demonstrating that the charge transfer between porphyrins and graphene is gate independent, and fixed at low temperature.

sample mobility [from $\mu=8000$ to $10000 \mathrm{~cm}^{2} /(\mathrm{V} \mathrm{s})$ near the Dirac point at $T=4.2 \mathrm{~K}$ ]. Hall plateaus are also better defined. These observations, along with the shift of the Dirac point to $V_{g} \simeq 0$, prove that porphyrins ionize and neutralize charged impurities on graphene or on the silicon substrate, and therefore decrease the disorder scattering.

This neutralization of graphene by ionization of the porphyrins is concurrent with the formation of singly occupied impurity states close to the Dirac point (distributed between the initial and final Dirac points). Whether or not these impurity states generate a magnetic moment, corresponding to the spin of the unpaired electron delocalized over the molecule, will depend on the energy of the impurity state, as well as on graphene's Fermi level, and thus gate voltage, as described by Uchoa et al. [6]. In the following, we present signatures of this magnetic moment and its gate-voltage dependence, detected via phase coherent transport measurements at low temperature.

\section{SIGNATURE OF MAGNETISM ON SAMPLES WITH NORMAL CONTACTS}

We first discuss three graphene samples, on the same $\mathrm{Si}$ substrate, with normal (nonsuperconducting) Ti(6nm)/Au(100 $\mathrm{nm})$ contacts. Two of them (Au1, Au2) were coated with porphyrins, another (Au3) not. We find (see Fig. 4) that at $1 \mathrm{~K}$ the two-terminal resistance in perpendicular field of the uncoated sample exhibits reproducible mesoscopic magnetoresistance fluctuations due to interference between all the coherent trajectories across the samples. The amplitude 

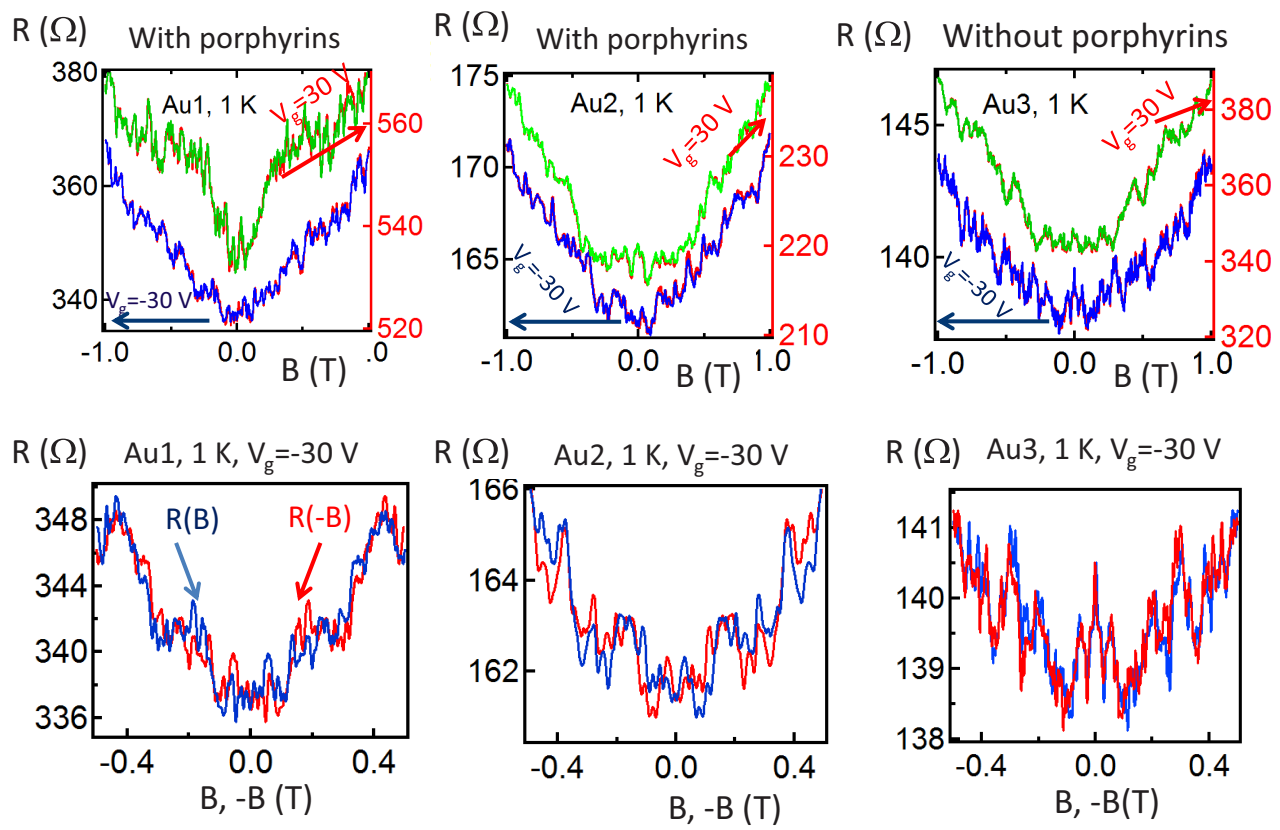

FIG. 4. Field asymmetry of the perpendicular magnetoresistance in porphyrins coated samples with Ti/Au electrodes at $1 \mathrm{~K}$. Out-of-plane magnetoresistance of three samples on the same chip is presented. Au1 and Au2 were coated with Pt-porphyrins, and Au3 was not. The resistance fluctuates with magnetic field in a reproducible way for all three samples (overlapping traces correspond to successive field sweeps). We find that the magnetoresistance curves of the coated samples are asymmetric. This asymmetry is better illustrated by plotting both the R(B) and R(-B) curves, see bottom panels. The asymmetry is clear for the samples coated with Porphyrins, Au1 and Au2, but not for the uncoated sample Au3.

of the conductance fluctuations is of the order of the conductance quantum. As for nonmagnetic mesoscopic samples, including graphene $[25,26]$, the curves of the uncoated samples are even functions of magnetic field, as expected for two probes measurements on nonmagnetic mesoscopic samples that obey time-reversal symmetry in zero field (see Fig. 4). By contrast, the magnetoresistance of the coated samples Au1 and $\mathrm{Au} 2$ contains an odd component, whose amplitude is of the order of $1 / 3$ of the even component. This indicates that time-reversal symmetry is broken in those samples in zero magnetic field, a fact that we attribute to macroscopic magnetic moments perpendicular to the sample plane, extending on spatial scales comparable to the phase coherence and thermal lengths. Similar effects have been observed in mesoscopic spin glasses [27]. This behavior must stem from frozen, long-range magnetic correlations within the ionized porphyrins, whose physical origin we discuss further in the paper. However, as shown in Fig. 5, this asymmetry of the magnetoresistance in a perpendicular field is undetectable at very low temperature $(100 \mathrm{mK})$. Instead, we find a strong asymmetric and hysteretic magnetoresistance in parallel field, with a large odd component whose amplitude and sign depend on gate voltage (see Ref. [28], Fig. S1). This asymmetric magnetoresistance in parallel field is not detectable at $1 \mathrm{~K}$. Thus it appears that the porphyrins' magnetic moment rotates from in-plane at $100 \mathrm{mK}$ to nearly out-of-plane at $1 \mathrm{~K}$. Since the average distance between ionized porphyrins is a few nanometers, our findings suggest a relatively long-ranged magnetic interaction mediated by graphene's conduction electrons or holes.

\section{SAMPLES WITH SUPERCONDUCTING CONTACTS}

We now turn to samples with superconducting electrodes (S/graphene/S junctions), to exploit the sensitivity of the Josephson current to magnetism. Whereas small effects are found in short junctions of length $L$ smaller than the superconducting coherence length $\xi_{S}$, (see samples Al1, Al2, and Al3 in supplementary materials), the most spectacular signatures of induced magnetism occur in long junctions for which $L \gg \xi_{S}$ or equivalently $\Delta \gg E_{\mathrm{Th}}$ (with $\Delta$ the superconducting gap, $E_{\mathrm{Th}}=\hbar D / L^{2}$ the Thouless energy and D the diffusion coefficient). This long-junction regime can be achieved either with large gap electrodes such as $\mathrm{Nb}$ (sample $\mathrm{Nb}$ ) or with very disordered samples with small $D$ (Ald1 and Ald2).

We first present a 1.2- $\mu \mathrm{m}$-long graphene sample connected to $\mathrm{Pd} / \mathrm{Nb}$ superconducting electrodes (8-nm $\mathrm{Pd} / 70-\mathrm{nm} \mathrm{Nb}$, sample $\mathrm{Nb}$ ), with $\xi_{S}=30 \mathrm{~nm}$. Figure 6 compares the gate voltage dependence of the differential resistance, at $100 \mathrm{mK}$, before and after porphyrins deposition. The resistance of the uncoated graphene junction is zero at low dc current, in highly doped regions (away from the Dirac point), both for hole and electron doping [Fig. 6(a)] [29,30]: this is the signature of a Josephson effect, a supercurrent running through the graphene because of the superconducting contacts. We previously reported that the amplitude of the maximal supercurrent, defined as the junction's critical current, is strongly depressed in the region of the Dirac point. We attribute that effect, observed only in long junctions, to specular reflections of the Andreev pairs on very low carrier density regions (puddles) where the local Fermi energy is smaller than the proximity induced superconducting gap (see Ref. [30] for a detailed 


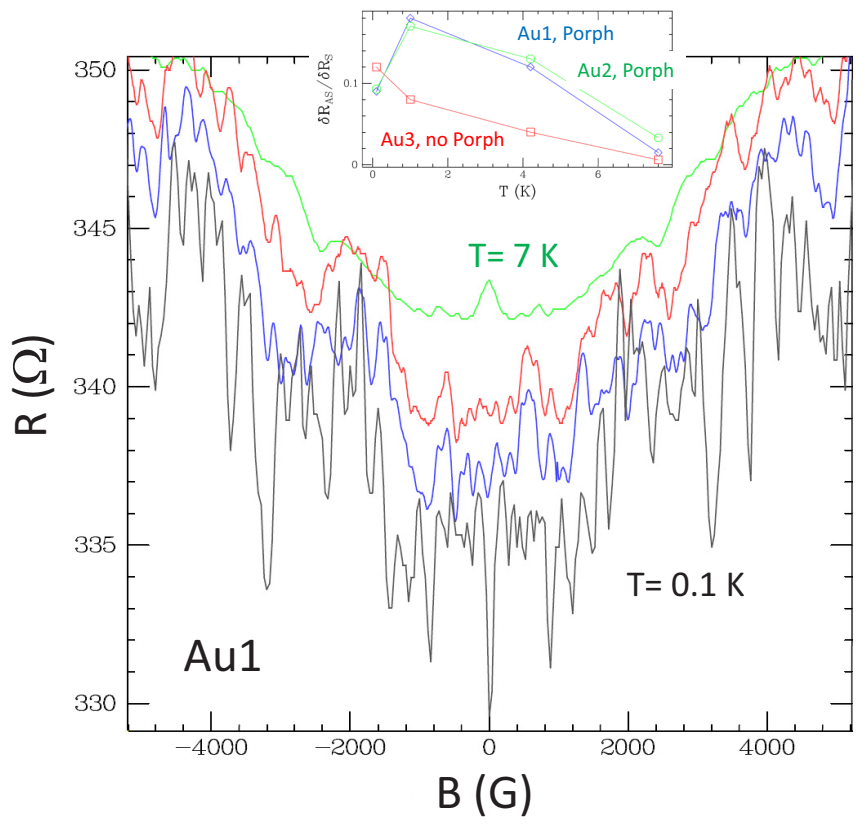

FIG. 5. $T$ dependence of asymmetry of resistance fluctuations in a perpendicular field for samples with Ti/Au electrodes. Resistance fluctuations in a perpendicular field for coated sample Au1, at 100 $\mathrm{mK}, 1 \mathrm{~K}, 4.2 \mathrm{~K}$, and $7 \mathrm{~K}$. The inset shows the asymmetry of the fluctuations [variance of the $R(B)-R(-B)$ curve], renormalized by the symmetric fluctuations [variance of $R(B)+R(-B)$ ] for all three samples (averaged over four curves for each sample), at $V_{g}=-30 \mathrm{~V}$. The asymmetry of the coated samples Au1 and Au2 is more than twice that of the uncoated sample Au3 at 1 and 4.2 $\mathrm{K}$. The relative asymmetry of the coated samples is maximal at $1 \mathrm{~K}$, when the moments are perpendicular to the graphene plane, and phase coherence is still achieved throughout the sample, so that the magnetoresistance fluctuations are sensitive to the magnetic moments. It is still visible at $4.2 \mathrm{~K}$. The loss of asymmetry at $7 \mathrm{~K}$ where mesoscopic fluctuations are reduced but still can be detected, can be attributed to the loss of long-range magnetic order. The loss of asymmetry at $100 \mathrm{mK}$ for the coated samples is explained by a moment in plane at low temperature. The slight low temperature increase of the asymmetry observed for the uncoated Au3 sample is due to the increase of mesoscopic telegraphic noise at low temperature observed on all graphene samples.

discussion of these findings). It is clear that at high doping $\left(V_{g}>20 \mathrm{~V}\right)$ the supercurrent exists for both electron and hole doping, as also shown in Fig. 6(c). In stark contrast to this bipolar Josephson effect through pristine graphene [Figs. 6(a) and 6(c)], we find that after deposition of the porphyrins, the Josephson current is enhanced in the hole doped region but suppressed in the electron-doped region. Only a dip in the differential resistance is visible at low current and high positive gate voltage, but no strictly zero resistance and no supercurrent [Fig. 6(b)]. Figure 6(c), which displays the critical current amplitude as a function of gate voltage, shows this strong asymmetry of the gate voltage dependence of the supercurrent after deposition of the porphyrins. The absence of supercurrent in the electron doped region was checked for gate voltages up to $60 \mathrm{~V}$.

We attribute this extinction of the critical current, at high positive gate voltage only, to an inhomogeneous magnetic field on graphene created by the staggered magnetism of the porphyrins. This destroys the constructive interferences between the Andreev pairs that carry the Josephson current. This sensitivity of the critical current to small magnetic perturbations is illustrated by the field dependence of the supercurrent in this junction, and its narrow [31] interference Fraunhoffer pattern [Fig. 6(d)]. It is clear that a field as small as a fraction of a Gauss can suppress the induced supercurrent through graphene, because of destructive interference between Andreev pairs diffusing across the graphene [32]. This explains how the porphyrin's magnetic spins, if they lead to correlated magnetic regions at positive gate voltage, can create an inhomogeneous magnetic flux sufficient to destroy the proximity effect, thereby leading to the observed unipolar supercurrent.

Another indication of gate-voltage-dependent magnetism comes from the magnetoresistance in a perpendicular magnetic field [Fig. 6(e)], which is a hysteretic function of field at $V_{g}>$ 0 , whereas there is no hysteresis for $V_{g}<0$. This absence of magnetic hysteresis and the large Josephson current in the hole doped region consistently indicate either a quenched magnetic moment for the porphyrins or a reduced exchange interaction between porphyrin's localised spins and the graphene holes. Moreover, the magnetic hysteresis in the electron doped region $\left(V_{g}>0\right)$ points to the formation of a magnetic order of the molecular spins, with partially oriented magnetic domains, generating a nonuniform magnetic field that is revealed by the magnetoresistance of graphene, and explains the suppressed Josephson current for this doping. No such effect is visible at high hole doping, which we attribute to the absence of magnetism in this gate voltage range. The asymmetry of induced magnetism with respect to the sign of gate voltage is striking. It was not observed in samples Au1 and Au2 discussed in the previous section but exists in samples with TiAl contacts discussed in the next section. This gate voltage "polarity" is probably strongly dependent on the initial doping and mobility of the graphene before deposition of porphyrins (see discussion below).

The signatures of magnetism are even more striking in data obtained on two last samples, Ald1 and Ald2, with Ti/Al contacts. These samples were initially highly electron-doped (Dirac point below $-40 \mathrm{~V}$, see inset of Fig. 7), as the result of oxygen plasma cleaning of the substrate before graphene deposition. Coating with porphyrins resulted in a shift of graphene's Dirac point to nearly zero gate voltage, despite the strong initial doping. This spectacular observation attests the huge capacity of charge transfer and neutralization of porphyrins. In this case, the porphyrins must act as acceptors of electrons to neutralize these initially electron-doped graphene samples. We expect a much higher concentration of ionized porphyrins in these samples compared to the ones discussed above, where the Dirac point shift did not exceed $15 \mathrm{~V}$. The high resistance at the Dirac point $(40 \mathrm{k} \Omega$ ) indicates a mobility at least 10 times smaller than the samples discussed previously. As a result we estimate a Thouless energy of the order of $4 \mu \mathrm{eV}$, much smaller than the superconducting contacts gap of the order of $200 \mu \mathrm{eV}$. The samples are thus also in the long-junction limit. We do not observe a supercurrent, at any gate voltage, but see up to a $75 \%$ drop of differential resistance at zero bias at high electron or hole doping. The low-field magnetoresistance of sample Ald1 for 

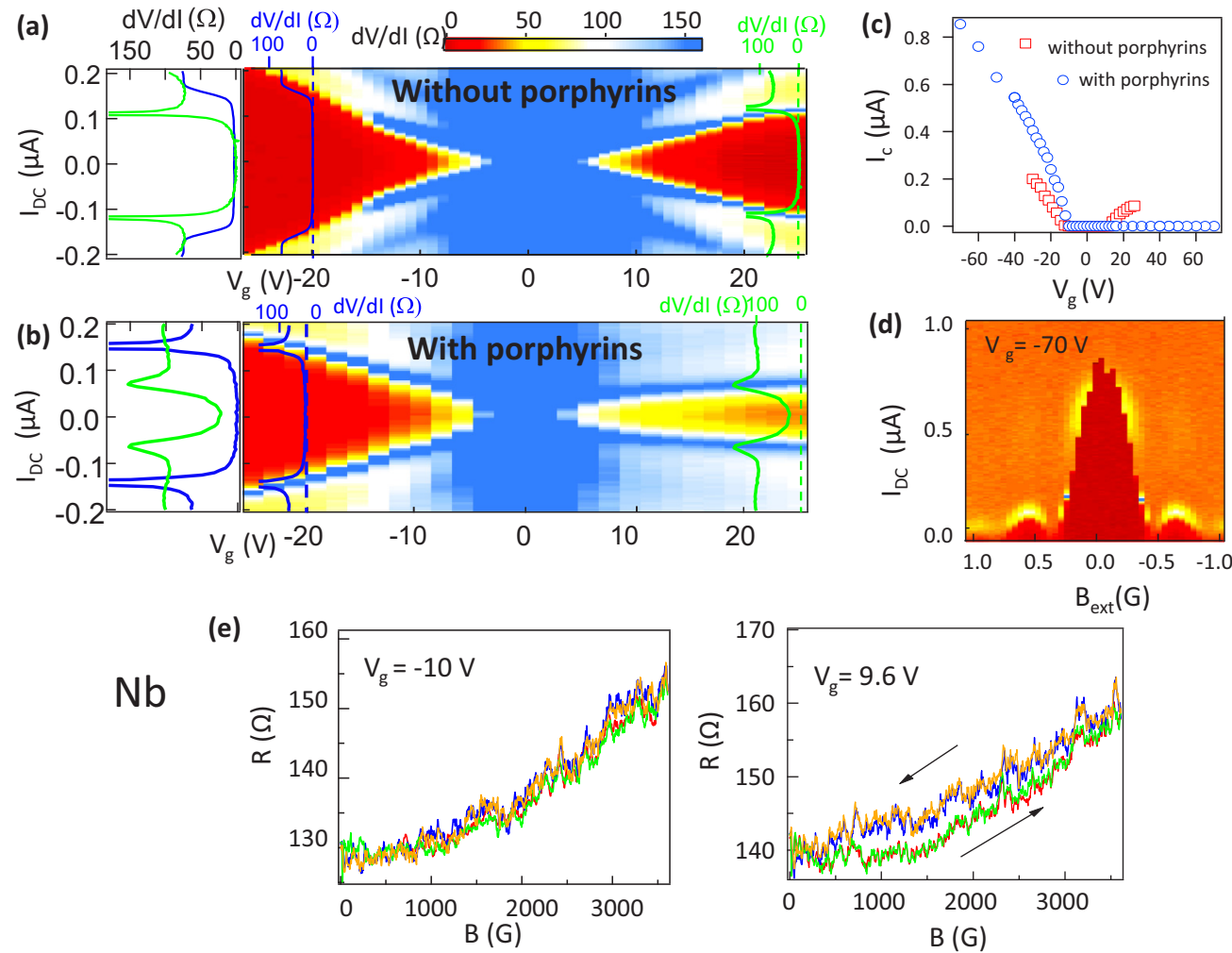

FIG. 6. Change from bipolar to unipolar induced superconductivity upon deposition of Pt-porphyrin on a sample with $\mathrm{Pd} / \mathrm{Nb}$ electrodes. (a) and (b) Color-coded differential resistance as a function of dc current ( $y$ axis) and gate voltage ( $x$ axis), measured with a small ac current added to the dc current. The dark red regions correspond to regions of zero differential resistance where a Josephson supercurrent runs through the S/graphene/S junction. Whereas the Josephson effect occurs symmetrically about the Dirac point on the pristine, uncoated sample [(a) $T=200 \mathrm{mK}]$, it only occurs on the hole doped side (negative $V_{G}$ ) on the sample covered with porphyrins [(b) $\left.T=100 \mathrm{mK}\right]$. The curves on the left of the color plots are the differential resistance curves as a function of dc current, at gate voltages symmetric with respect the Dirac point. (c) Change from bipolarity to unipolarity upon coating, revealed by the variations with gate voltage of the critical current $I_{c}$, i.e., highest dc current for which the differential resistance is zero. Before (red squares, bipolar) and after (blue circles, unipolar) porphyrin deposition. (d) Differential resistance as a function of dc current and external magnetic field (perpendicular to the graphene sheet), for the graphene with porphyrin molecules, at $V_{G}=-70 \mathrm{~V}$ revealing a Fraunhofer pattern. (e) Hysteresis in the graphene's magnetoresistance after porphyrin deposition, at $100 \mathrm{mK}$, with a field direction perpendicular to the graphene plane. The hysteresis is quite large at positive gate voltage (electron doping, right curves) and negligible (at least an order of magnitude smaller) for hole doping (left curves), confirming the existence of a magnetic order that suppresses the supercurrent for electron doping.

different gate voltages is shown in Fig. 7. Whereas at high doping the magnetoresistance dips at zero field and displays oscillations typical of a Fraunhofer interference pattern, the magnetoresistance is clearly peaked at $B=0$ close to the Dirac point, and displays jumps at specific magnetic fields. Field asymmetry and hysteresis are observed for all gate voltages, indicating that this magnetoresistance is due to correlated magnetic regions in the graphene sample. This magnetism is however asymmetric with respect to the Dirac point. It is maximum for negative gate voltage and decreases for positive gate voltage. This is illustrated in the inset of Fig. 7 showing the variance $\delta R_{A S} / R$ of the odd component of the magnetoresistance renormalized to the average total resistance as a function of the gate voltage. We attribute this asymmetry (opposite to $\mathrm{Nb}$ sample case) to the initial strong electron doping of the samples.

We have conducted similar investigations in short-junction samples (see Ref. [28]). Whereas porphyrins coating does not suppress the critical current at zero field, we do find distortions of the Fraunhofer patterns, as well as important asymmetries with respect to the electron/hole doping in finite magnetic field (at the edges of the Fraunhofer lobes).

\section{DISCUSSION}

We attribute the doping-dependent asymmetries, hysteresis in magnetic field, and doping-dependent suppression of Josephson current, to a gate-voltage-dependent magnetism due to the ionized porphyrin molecules. Moreover, these results imply the existence of magnetic domains in the samples, and therefore interactions between the individual magnetic moments of the porphyrins. In addition, the data are consistent with magnetic moments preferentially aligned, at very low temperatures, in the molecules' plane, parallel to the graphene layer. This is evidenced by the strong hysteretic odd component of the in-plane magnetoresistance at low temperature in the graphene samples connected to normal electrodes (see supplementary materials). By contrast, when graphene is connected to superconducting electrodes, the hysteresis occurs also for perpendicular field. We explain this by the focusing and 


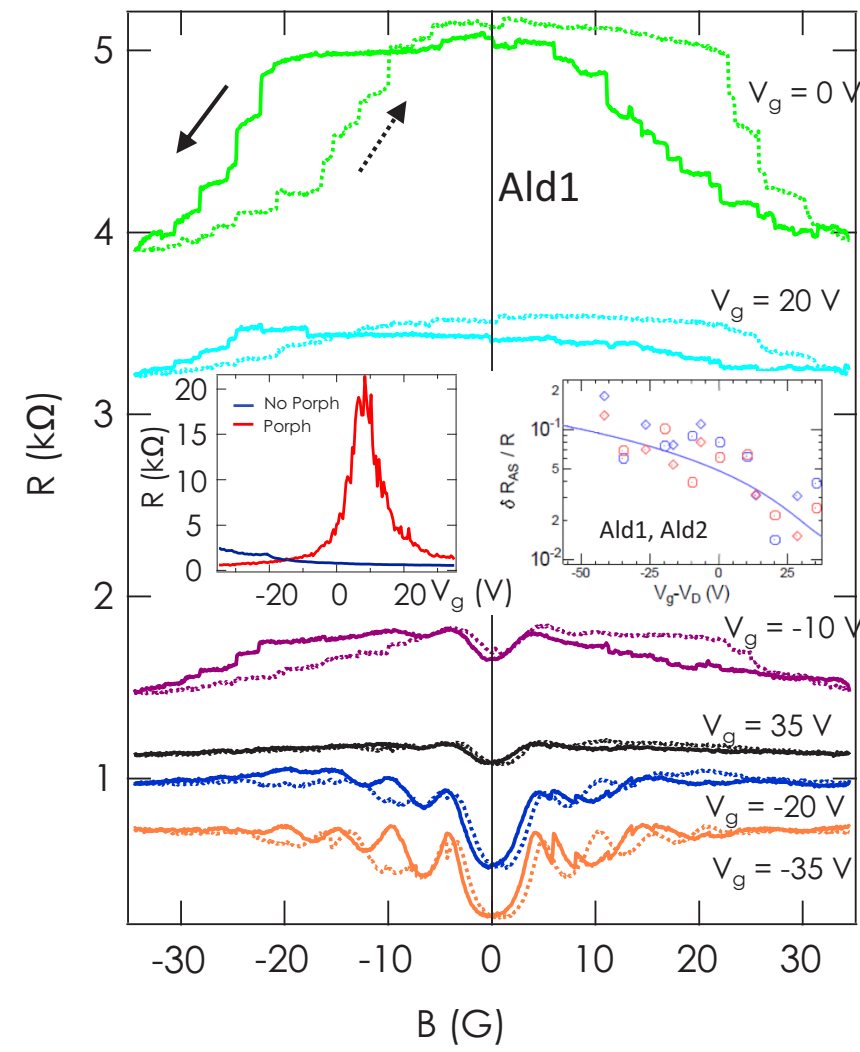

FIG. 7. Proximity induced superconductivity (Ti/Al contacts) in a low mobility long-junction sample coated with porphyrins. Magnetoresistance of sample Ald1 at different gate voltages. Up and down magnetic field sweeps are respectively dashed and solid lines, the sweep directions are indicated by arrows. The hysteresis at low doping is accompanied by many jumps corresponding to strongly correlated magnetic domains. (Left inset) Gate dependence before (blue) and after (red) porphyrin deposition, showing the striking neutralization power of porphyrin molecules on graphene and, in this case, the property of porphyrins to act as electron acceptors. (Right inset) Gate voltage dependence of the antisymmetric component of the magnetoresistance $\delta R_{A S}$ rescaled to the mean resistance R, of the samples Ald1 (diamonds) and Ald2 (circles). Red and blue points correspond to up and down magnetic field sweeps, respectively.

bending of the field lines by the superconducting electrodes, so that a perpendicular external field leads to a local field on the graphene sheet that contains both parallel and perpendicular components. Finally, we have also checked that there is no magnetic hysteresis or asymmetry both with normal or superconducting electrodes in the absence of porphyrins (see Ref. [28]).

The physics of magnetic impurities on graphene has led to a variety of interesting theoretical predictions specific to the band structure of graphene. Unlike metals, the exchange coupling in graphene is expected to be controlled by gating [3]. This effect has two important consequences. First, the amplitude of the magnetic moment of an individual impurity should strongly depend on gate voltage, causing a tunable Kondo effect. Depending on the relative energy of the impurity level with respect to the Dirac point, its magnetic state could be highly asymmetric with gate voltage [6] with impurities which have a nonzero magnetic moment for only one sign of gate voltage. Second, the long-range magnetic Rudderman, Kittel, Kasuya, and Yosida (RKKY) interactions mediated by the carriers should also be gate voltage dependent. Such interactions have been investigated theoretically and numerically by several groups $[3,33]$. Characteristic coupling energies in the Kelvin range are predicted between spins $1 / 2$ a few nanometers apart. The magnetic hysteresis we observe could then be explained by a spin glass type of order taking place at low temperature.

Interestingly, it was recently shown [34] that exchange interactions in proximity induced superconducting graphene are enhanced on the scale of the coherence length compared to the normal state and acquire an antiferromagnetic sign in the vicinity of the Dirac point. Our results show that porphyrins coated graphene offers the possibility to explore this physics as well. Of course extrapolating these theoretical findings on the exchange coupling between localized spins on graphene to the case of Pt-porphyrins, where each spin is localized over the entire nanometer sized molecule is still a challenge [35].

At this stage, let us summarize our understanding of the non-systematic occurrence of the gate-voltage polarity in the magnetism we report. The gate-voltage polarity of magnetism is explained by the initial doping of graphene before porphyrins deposition, and the subsequent pinning of the HOMO or LUMO level of the porphyrins upon coating and charge transfer. This leads to impurity states on average below the Dirac point for initially electron doped graphene $(\mathrm{Nb}$ sample), and above the Dirac point for initially hole-doped graphene (sample Ald1 and Ald2). As shown in Ref. [6], there is a limited gate range over which these impurity states are singly occupied and thus magnetic, and the range differs for both cases. The range nonetheless extends around the impurity levels energies, explaining why the magnetism should be more pronounced in positive gate voltage in the $\mathrm{Nb}$ sample, and negative gate voltage for the Ald1 and Ald2 samples. In contrast, samples with initially relatively broad Dirac peaks centered close to zero gate voltage due to both electron and hole pockets (such as Au1 and Au2 samples), are bound to have impurity levels both above and below the Dirac point, in a relatively wide range, and consequently magnetism is expected for both positive and negative gate voltage.

In conclusion, this gate voltage-dependent long-range order of magnetic porphyrins mediated by graphene's carriers is of great potential interest and motivates further investigations of different porphyrin species, including metal free porphyrins or ones with a magnetic atom such as Fe or Co. Our findings show that it is possible to control the number of magnetic porphyrins by their degree of ionization. This suggests interesting possibilities such as controling the magnetism by a gate voltage quench of graphene, or with a controlled concentration of charged defects. Finally, we provide a new route for inducing gate-dependent long-range magnetism in graphene without destroying the sample mobility. 


\section{ACKNOWLEDGMENTS}

We acknowledge ANR Supergraph and CNRS for funding, Juan Manuel Aguilar, Alexei Chepelianskii, Richard Deblock, Andrew Mayne, Claude Pasquier, Lau- rent Simon, and Alberto Zobelli for helpful discussions, and M. Feigelmann for suggesting that the suppressed proximity effect could be due to the molecules' magnetism.
[1] A. H. Castro Neto, F. Guinea, N. M. R. Peres, K. S. Novoselov, and A. K. Geim, The electronic properties of graphene, Rev. Mod. Phys. 81, 109 (2009); M. Monteverde, C. OjedaAristizabal, R. Weil, K. Bennaceur, M. Ferrier, S. Guéron, C. Glattli, H. Bouchiat, J. N. Fuchs, and D. L. Maslov, Transport and Elastic Scattering Times as Probes of the Nature of Impurity Scattering in Single-Layer and Bilayer Graphene, Phys. Rev. Lett. 104, 126801 (2010).

[2] F. Schedin, A. K. Geim, S. V. Morozov, E. W. Hill, P. Katsnelson Blake, K. S. Novoselov, Detection of individual gas molecules adsorbed on graphene, Nat. Mater. 6, 652 (2007).

[3] V. N. Kotov, B. Uchoa, V. M. Pereira, F. Guinea, A. H. Castro Neto, Electron-electron interactions in graphene: Current status and perspectives, Rev. Mod. Phys. 84, 1067 (2012).

[4] C. Weeks, J. Hu, J. Alicea, M. Franz, and R. Wu, Engineering a Robust Quantum Spin Hall State in Graphene via Adatom Deposition, Phys. Rev. X 1, 021001 (2011).

[5] Mahmoud M. Asmar and Sergio E. Ulloa, Spin-Orbit Interaction and Isotropic Electronic Transport in Graphene, Phys. Rev. Lett. 112, 136602 (2014)

[6] Bruno Uchoa, Valeri N. Kotov, N. M. R. Peres, and A. H. Castro Neto, Localized Magnetic States in Graphene, Phys. Rev. Lett. 101, 026805 (2008).

[7] K. Pi, K. M. McCreary, W. Bao, W. Han, Y. F. Chiang, Y. Li, S.-W. Tsai, C. N. Lau, and R. K. Kawakami, Electronic doping and scattering by transition metals on graphene, Phys. Rev. B 80, 075406 (2009).

[8] M. Alemani, A. Barfuss, B. Geng, C. Girit, P. Reisenauer, M. F. Crommie, F. Wang, A. Zettl, and F. Hellman, Effect of gadolinium adatoms on the transport properties of graphene, Phys. Rev. B 86, 075433 (2012).

[9] A. Candini, S. Klyatskaya, M. Ruben, W. Wernsdorfer, and M. Affronte, Graphene spintronic devices with molecular nanomagnets, Nano Lett. 11, 2634 (2011).

[10] Jayakumar Balakrishnan, Gavin Kok Wai Koon, Ahmet Avsar, Yuda Ho, Jong Hak Lee, Manu Jaiswal, Seung-Jae Baeck, JongHyun Ahn, Aires Ferreira, Miguel A. Cazalilla, Antonio H. Castro Neto, and Barbaros Ozyilmaz, Giant spin hall effect in graphene grown by chemical vapor deposition, Nat. Commun. 5, 4748 (2014).

[11] R. R. Nair, M. Sepioni, I-Ling Tsai, O. Lehtinen, J. Keinonen, A. V. Krasheninnikov, T. Thomson, A. K. Geim and I. V. Grigorieva, Spin-half paramagnetism in graphene induced by point defects, Nat. Phys. 8, 199 (2012).

[12] A. Candini, C. Alvino, W. Wernsdorfer, and M. Affronte, Hysteresis loops of magnetoconductance in graphene devices, Phys. Rev. B 83, 121401(R) (2011).

[13] Kathleen M. McCreary, Adrian G. Swartz, Wei Han, Jaroslav Fabian, and Roland K. Kawakami, Magnetic Moment Formation in Graphene Detected by Scattering of Pure Spin Currents, Phys. Rev. Lett. 109, 186604 (2012).
[14] A. Mayne et al. (unpublished).

[15] P. Chen, O. S. Finikova, A. Ou, S. A. Vinogradov, and K. M. Kadish, Electrochemistry of platinum(II) porphyrins: Effect of substituents and $\pi$-extension on redox potentials and site of electron transfer, Inorg. Chem. 51, 6200 (2012).

[16] M. B. Lundeberg, R. Yang, J. Renard, and J. A. Folk, DefectMediated Spin Relaxation and Dephasing in Graphene, Phys. Rev. Lett. 110, 156601 (2013).

[17] G. Clavé, G. Delport, C. Roquelet, J.-S. Lauret, E. Deleporte, F. Vialla, B. Langlois, R. Parret, C. Voisin, P. Roussignol, B. Jousselme, A. Gloter, O. Stephan, A. Filoramo, V. Derycke, and S. Campidelli, Functionalization of Carbon Nanotubes through Polymerization in Micelles: A Bridge between the Covalent and Noncovalent Methods, Chem. Mater. 25, 2700 (2013); PtII tetrakis (4-carboxyphenyl)-porphyrin ( $15 \mathrm{mg}, 10.8 \mu \mathrm{mol}$ ) was dissolved in NMP (4 ml). S-acetylcysteamine hydrochloride $(13.54 \mathrm{mg}$, $86 \mu \mathrm{mol})$, benzotriazol-1-yl-oxytripyrrolidinophosphonium hexafluorophosphate (PyBOP) reagent $(44.7 \mathrm{mg}, 86 \mu \mathrm{mol})$ and dry DIEA (30 $\mu l, 172 \mu \mathrm{mol})$ were sequentially added and the resulting reaction mixture was stirred at room temperature for $4 \mathrm{~h}$. The reaction was checked for completion by TLC $\left(\mathrm{CH}_{2} \mathrm{Cl}_{2} / \mathrm{MeOH}, 96: 4\right.$, v/v). Thereafter, the crude was diluted with $\mathrm{AcOEt}(40 \mathrm{ml})$, washed by aq. $10 \%$ citric acid $(40 \mathrm{ml})$, aq. sat. $\mathrm{NaHCO}_{3}(40 \mathrm{ml})$ and brine $(40 \mathrm{ml})$. The organic layer was dried over $\mathrm{Na}_{2} \mathrm{SO}_{4}$, filtrated and evaporated to dryness. The resulting residue was purified by chromatography on a silica gel, eluent $\left(\mathrm{CH}_{2} \mathrm{Cl}_{2} / \mathrm{MeOH}\right.$ : $100 / 0$ to $\left.97 / 3\right)$, yielding porphyrins as a brown-orange solid $(9.9 \mathrm{mg}, 7.1 \mu \mathrm{mol}$, yield $=66 \%) .{ }^{1} \mathrm{H}$ NMR $\left(300 \mathrm{MHz}, \mathrm{CDCl}_{3}\right): \delta=3.64(\mathrm{~s}, 3 \mathrm{H}), 3.85(t, J=5.1$ $\mathrm{Hz}, 2 \mathrm{H}), 4.16-4.19$, (m, 2H), 8.09 (s, 8H), 8.15-8.23 (m, 12H), 8.70 (s, 4H). MS (MALDI-TOF) $\mathrm{m} / z=1387.26[\mathrm{M}]^{+}$, calculated for $\mathrm{C}_{64} \mathrm{H}_{56} \mathrm{~N}_{8} \mathrm{O}_{8} \mathrm{~S}_{4}$ Pt: $1387.28 \mathrm{~g} / \mathrm{mol}$.

[18] L. Scudiero, Dan E. Barlow, Ursula Mazur, and K. W. Hipps, Scanning tunneling microscopy, orbital-mediated tunneling spectroscopy, and ultraviolet photoelectron spectroscopy of metal(II) tetraphenylporphyrins deposited from vapor, J. Am. Chem. Soc. 123, 4073 (2001).

[19] D. Tasis, N. Tagmatarchis, N. Bianco, and M. Prato, Chemistry of carbon nanotubes, Chem. Rev. 106, 1105 (2006).

[20] D. S. Hecht et al., Bioinspired detection of light using a porphyrin-sensitized single-wall nanotube field effect transistor, Nano Lett. 6, 2031 (2006).

[21] M. S. Choi et al., Gate-dependent photoconductivity of single layer graphene grafted with metalloporphyrin molecules, Appl. Phys. Lett. 100, 163116 (2012).

[22] P. Checcolia et al., Tetra-phenyl porphyrin based thin film transistors, Synth. Metals 138, 261 (2003).

[23] G. Sedghi et al., Long-range electron tunneling in oligoporphyrin molecular wires, Nat. Nanotechnol. 6, 517 (2011). 
[24] Deqi Wang, Xinfei Liu, Le He, Yadong Yin, Di Wu, and Jing Shi, Manipulating graphene mobility and charge neutral point with ligand-bound nanoparticles as charge reservoir, Nano Lett. 10, 4989 (2010).

[25] Mark B. Lundeberg and Joshua A. Folk, Spin-resolved quantum interference in graphene, Nat. Phys. 5, 894 (2009)

[26] C. Ojeda-Aristizabal, M. Monteverde, R. Weil, M. Ferrier, S. Guéron, and H. Bouchiat, Conductance Fluctuations and Field Asymmetry of Rectification in Graphene, Phys. Rev. Lett. 104, 186802 (2010).

[27] P. G. N. de Vegvar, L. P. Levy, and T. A. Fulton, Conductance Fluctuations of Mesoscopic Spin Glasses, Phys. Rev. Lett. 66, 2380 (1991).

[28] See Supplemental Material at http://link.aps.org/supplemental/ 10.1103/PhysRevB.93.045403 for in- and out-of-plane magnetotransport in samples with normal $(\mathrm{Ti} / \mathrm{Au})$ contacts, and out-of-plane magnetotransport of S/graphene/S junctions with $\mathrm{Ti} / \mathrm{Al}$ contacts, including an especially disordered graphene sample.

[29] All reported experiments with supercurrents induced through graphene found a bipolar proximity effect. The first report is B. Heersche Hubert, P. Jarillo-Herrero, B. Oostinga Jeroen, M. K. Vandersypen Lieven, and F. Morpurgo Alberto, Bipolar supercurrent in graphene, Nature (London) 446, 56 (2007). Other references are given in Ref. [30].
[30] K. Komatsu, C. Li, S. Autier-Laurent, H. Bouchiat, and S. Guéron, Superconducting proximity effect in long superconductor/graphene/superconductor junctions: From specular Andreev reflection at zero field to the quantum Hall regime, Phys. Rev. B 86, 115412 (2012).

[31] A flux quantum through the area of the sample $\left(1.2 \times 12 \mu \mathrm{m}^{2}\right)$ corresponds to a field of 3 Gauss, which is much larger than the value of 0.4 Gauss measured experimentally. We understand this effect (also reported in many other papers on the proximity effect in 2D materials) as due to the focusing of field lines between the two massive $\mathrm{Nb}$ superconducting electrodes whose width is equal to $5 \mu \mathrm{m}$.

[32] F. Chiodi, M. Ferrier, S. Guéron, J. C. Cuevas, G. Montambaux, F. Fortuna, A. Kasumov, H. Bouchiat, Geometry-related magnetic interference patterns in long SNS Josephson junctions, Phys. Rev. B 86, 064510 (2012).

[33] A. M. Black-Schaffer, RKKY coupling in graphene, Phys. Rev. B 81, 205416 (2010).

[34] N. Y. Yao, L. I. Glazman, E. A. Demler, M. D. Lukin, and J. D. Sau, Enhanced Anti-Ferromagnetic Exchange Between Magnetic Impurities in a Superconducting Host, Phys. Rev. Lett. 113, 087202 (2014).

[35] Christian F. Hermanns, Kartick Tarafder, Matthias Bernien, Alex Krüger, Yin-Ming Chang, Peter M. Oppeneer, and Wolfgang Kuch, Magnetic coupling of Porphyrin molecules through graphene, Adv. Mater. 25, 3473 (2013). 